\title{
Influenza em crianças: o que há de novo?
}

\section{Influenza in children: what is new?}

Lucia Ferro Bricks ${ }^{1}$, Carla Magda Allan Santos Domingues ${ }^{2}$, Telma Regina Marques Pinto Carvalhanas ${ }^{3}$, Sirlene de Fátima Pereira $^{2}$, Jose Cassio de Moraes ${ }^{4}$

1. Sanofi Pasteur Divisão Vacinas do Grupo Sanofi, Brasil, 2. Programa Nacional de Imunizações do Ministério da Saúde do Brasil, 3. Centro de Vigilância Epidemiológica (CVE), Divisão Respiratórias da Secretaria de Saúde do Estado de São Paulo, Brasil, 4. Faculdade de Ciências Médicas da Santa Casa de São Paulo, Brasil.

\begin{abstract}
Resumo
Introdução: A influenza afeta pessoas de todas as idades, causando substancial impacto em Saúde Pública. No Brasil, em 2014, a vacinação de crianças foi ampliada, abrangendo todas as crianças de 6 a 60 meses de idade. Objetivo: Analisar as informações da literatura que justificam a ampliação da vacinação na faixa etária pediátrica. Métodos: Foram identificados e analisados os artigos publicados no PUBMED e SCIELO, assim como as informações disponíveis nos sites oficiais da OMS, OPAS, CDC, ECDC e do Ministério da Saúde do Brasil, utilizando as palavras-chave influenza, vacinas, crianças, no período de 01/01/2003 a 30/04/2014 e consultadas as fontes relevantes mencionadas nos artigos. Resultados: Crianças menores de cinco anos apresentam as mais elevadas taxas de infecção por influenza e são as principais transmissoras do vírus para seus contatos na família e na escola. Nessa faixa etária, são comuns as complicações da influenza, como otite média aguda, crises de broncoespasmo e pneumonia. 0 número de consultas ambulatoriais e em serviços de emergência, assim como as hospitalizações são substanciais, assim como o absenteísmo dos pais ao trabalho para cuidar de crianças doentes, acarretando enormes perdas econômicas. Existem evidências de que a vacinação além de proteger as crianças vacinadas (proteção direta), reduz a carga da doença entre seus contatos (proteção indireta). Conclusões: A ampliação da vacinação de crianças pode reduzir substancialmente a morbidade e a mortalidade em toda a população e gerar economia para a família e a sociedade.
\end{abstract}

Palavras-chave: Influenza. Vacinas. Imunização, crianças. Epidemiologia.

\begin{abstract}
Introduction: Influenza affects people of all ages, causing a material impact on Public Health. In Brazil, children vaccination has been expanded in 2014, to cover all children aged 6 to 60 months. Objective: This review aims at assessing information available in the literature that supports the expansion of vaccination coverage in the pediatric age group. Methods: Material published in PUBMED and SCIELO, has been identified and reviewed, as well as the information available in the WHO, PAHO, CDC, ECDC official sites and in that of the Health Ministry of Brazil, for the period 01/01/2003 to 04/30/2014 using the key-words influenza, vaccines, children Relevant sources mentioned in those articles have also been reviewed. Results: Children under five years of age exhibit the highest influenza infection rates and are the main virus transmitters to their family and school contacts. Complications of influenza, such as acute otitis media, bronchospasm crises and pneumonia are common in this age group. The number of outpatient and emergency service visits, as well as hospitalizations, are substantial, just as work absenteeism of parents who must take care of sick children, causing huge economic losses. There are evidences that vaccination, besides protecting the vaccinated children (direct protection), reduces the disease load among their contacts (indirect protection). Conclusions: The expansion of children vaccination coverage may substantially reduce morbidity and mortality in whole the population and generates savings for the family and for society.
\end{abstract}

Keywords: Influenza. Vaccines. Immunization, children. Epidemiology.

\section{INTRODUÇÃO}

De acordo com a Organização Mundial da Saúde, estima-se que o vírus influenza acometa 5 a $15 \%$ da população, causando 3 a 5 milhões de casos graves e 250.000 a 500.000 mortes todos os anos ${ }^{1}$. A influenza afeta pessoas de todas as idades e, durante as epidemias sazonais, causa substancial impacto em Saúde Pública e na economia devido ao aumento no número de consultas, hospitalizações, faltas à escola e ao trabalho e gastos para tratar a doença ${ }^{2}$. A vacinação anual contra a influenza é a medida mais efetiva para prevenir a doença e suas complicações e é indicada por diversas autoridades de saúde, embora os grupos prioritários para vacinação variem entre os diferentes países ${ }^{1,3-11}$. Em 2014, a vacinação contra influenza foi ampliada no Brasil, sendo estendida de 6 a 24 meses para 6 a 60 meses $^{10}$. Este artigo tem como objetivo analisar as informações mais atuais da literatura que justificam a ampliação da vacinação na faixa etária pediátrica.

\section{MÉTODOS}

Foram identificados e analisados os artigos publicados no PUBMED e SCIELO, assim como as informações disponíveis nos sites oficiais da Organização Mundial da Saúde (OMS)

Correspondência: Lucia Ferro Bricks. Diretora Médica Influenza Sanofi Pasteur, Brasil. Av. Major Sylvio de Magalhães Padilha, no 5.200 - Edifício Atlanta, Morumbi, CEP: 05677-000 - São Paulo, SP, Brasil. E-mail: lfbricks@gmail.com

Conflito de interesse: Bricks LF. Declaro ser empregada da Sanofi Pasteur, onde exerço atualmente a função de Diretora Médica para Influenza na América Latina

Recebido em: 14 Jun 2014; Revisado em: 28 Jun 2014; Aceito em: 22 Jul 2014. 
Organização Pan-americana da Saúde (OPAS), Centro de Controle de Doenças (CDC) dos Estados Unidos da América (EUA), Centro de Controle de Doenças da Europa (ECDC) e do Ministério da Saúde do Brasil, utilizando as palavras-chave influenza, vacinas e crianças, no período de 01/01/2003 a $30 / 04 / 2014$ nos idiomas inglês, português e espanhol. Desde que a vacina influenza foi recomendada para crianças saudáveis nos EUA, os autores têm acompanhado a literatura sobre o tema, selecionando os artigos mais relevantes publicados no PUBMED e SCIELO. Após a pandemia de 2009, identificamos 217 artigos de revisão utilizando as palavras-chave influenza e crianças no PUBMED. Destes, excluímos 99 que tinham como objetivo analisar os seguintes tópicos: tratamento da influenza, vacinas não indicadas para crianças, vacinas em desenvolvimento, resposta imune ou eventos adversos de vacinas, uso de vacinas em grupos de risco e não em crianças saudáveis. Após revisar as mais recentes recomendações para uso da vacina influenza em crianças saudáveis encontradas nos sites da OMS, PAHO, CDC e Ministério da Saúde do Brasil, assim como artigos que subsidiaram a inclusão da vacina em crianças nos EUA, Canadá, Austrália, Europa e no Brasil, foram analisados 141 textos para esta revisão.

\section{Agente etiológico e transmissão}

$A$ doença pode ser causada pelos vírus influenza $A, B$ e $C^{2,7}$. Os vírus $A$ e $B$ apresentam maior importância clínica; estima-se que, em média, as cepas A causem $75 \%$ das infecções, mas em algumas temporadas, ocorre predomínio das cepas $\mathrm{B}^{12-23}$, que parecem ter maior impacto em crianças, adolescentes e adultos jovens ${ }^{14,22,23}$. Em 2013, por exemplo, mais da metade das mortes registradas em crianças nos EUA foram causadas pela cepa $\mathrm{B}^{8}$.

A presença de imunidade prévia reduz as chances de infecção, mas a imunidade a um subtipo $A$ ou linhagem $B$ confere pouca ou nenhuma proteção contra novas variantes ${ }^{2,24,25}$, por isso, numa mesma temporada de influenza, podem ocorrer infecções por mais de um tipo ou subtipo de vírus influenza. Dependendo da virulência das cepas circulantes, o número de hospitalizações e mortes aumenta substancialmente, não apenas por infecção primária, mas também devido às infecções secundárias por bactérias ${ }^{26-31}$. A gravidade da doença é maior quando surgem cepas pandêmicas, para as quais a população tem pouca ou nenhuma imunidade ${ }^{2,3,29-32}$, mas as cepas sazonais também podem causar altas taxas de hospitalizações e mortes durante algumas estações ${ }^{27,33}$. A cepa $\mathrm{A}(\mathrm{H} 1 \mathrm{~N} 1)$ pdm09, responsável pela pandemia de 2009, atualmente é considerada uma cepa sazonal. Em 2013, causou substancial número de mortes no Brasil, com predomínio na faixa etária de 29-59 $\operatorname{anos}^{14}$ e, na temporada 2013/2014, tem causado grande número de mortes no México ${ }^{34}$ e de hospitalizações nos EUA ${ }^{35}$.

\section{Transmissão do vírus influenza}

A transmissão ocorre principalmente por meio do contato com partículas eliminadas por pessoas infectadas ou mãos e objetos contaminados por secreções ${ }^{2,3,7}$. Estima-se que uma pessoa infectada seja capaz de transmitir o vírus para até dois contatos não imunes ${ }^{36}$.
Pessoas infectadas podem disseminar os vírus até 1,8 metros de distância e o uso de máscaras comuns é insuficiente para proteger contra infecção por partículas pequenas (aerossóis), mesmo na ausência de procedimentos invasivos ${ }^{37,38}$.

A excreção viral nas secreções nasais usualmente surge 24 horas antes do início dos sintomas; é máxima nos primeiros três dias após início das manifestações clínicas, coincidindo com a piora clínica e aumento da temperatura ${ }^{39}$. A maioria dos adultos é capaz de infectar outras pessoas de um dia antes do início dos sintomas, até 5 a 7 dias após adoecer. Crianças podem excretar os vírus por até duas semanas e os imunocomprometidos durante meses. ${ }^{2}$ A transmissão é muito alta em ambiente domiciliar, creches, escolas e em ambientes fechados ou semifechados, dependendo não apenas da infectividade das cepas, mas também do número e da intensidade dos contatos entre pessoas de diferentes faixas etárias ${ }^{11,16,29,40-47}$.

Ambas as cepas, A e B, têm sido identificadas em surtos registrados em hospitais ${ }^{48-50,}$ em militares ${ }^{51-53}$ e trabalhadores de refinarias petróleo ${ }^{54}$, após catástrofes, como terremotos ${ }^{55}$, em turistas e tripulantes de navios ${ }^{56-61}$ e aviões ${ }^{62,63}$ e entre pessoas que frequentam locais aglomerados, como festivais de música, eventos religiosos ou esportivos ${ }^{64-67}$.

Os vírus influenza mantêm capacidade de infecção por mais de 24 horas em superfícies lisas não porosas e podem permanecer infectantes durante meses em água doce, dependendo das condições de temperatura. O aumento da salinidade reduz a capacidade de infecção, enquanto a queda de temperatura está associada ao aumento na sobrevida dos vírus influenza na água. A sobrevida e a capacidade de infecção podem ser mantidas por mais de um ano, dependendo dessas condições ${ }^{68}$.

Eventualmente, o ser humano pode adquirir a doença em contato próximo com animais, como aves e porcos, mesmo assintomáticos $2,3,69,70$. A gravidade dessas infecções é variável. A variante $A(H 3 N 2) v$ identificada recentemente nos EUA tem causado doença leve e autolimitada ${ }^{71}$, enquanto as cepas $A(H 5 N 1)$ e $A(H 7 N 9)$ apresentam alta taxas de letalidade ${ }^{72,73}$.

As crianças com idade entre um e cinco anos são as principais fontes de transmissão dos vírus na família e na comunidade ${ }^{29,42-44,74-81}$. Em geral, as epidemias de influenza são anunciadas pelo aumento na busca de atendimentos médicos por crianças com febre..$^{23,74,75} \mathrm{Em}$ um estudo que incluiu 313 escolares de 216 famílias, para cada 100 crianças acompanhadas, a síndrome gripal, principal forma de apresentação da influenza, foi responsável por 28 episódios de doença respiratória, por 20 dias de absenteísmo dos pais ao trabalho e por 22 casos secundários na família ${ }^{76}$.

\section{Manifestações clínicas}

O período de incubação dos vírus influenza varia entre um e quatro dias. Os sinais e sintomas da doença são muito variáveis $^{2,3}$. A maioria das infecções é assintomática ${ }^{82-84}$. A síndrome gripal, forma de apresentação clínica mais comum 
da influenza, caracteriza-se pelo súbito aparecimento de febre, tosse, dor de garganta, cefaleia, mialgia e fadiga; também pode ser causada por diversos outros agentes infecciosos ${ }^{2,3}$. Durante a época de maior circulação (sazonalidade), os vírus influenza são identificados em até $30 \%$ dos casos de síndrome gripal, ${ }^{3,85}$ principal causa de consulta média em serviços ambulatoriais e um aumento paralelo nas taxas de hospitalização por pneumonia e síndrome respiratória aguda grave ${ }^{28,76,84,86-90}$; por isso, os registros de pneumonia e influenza são computados em conjunto ${ }^{33}$.

Deve-se ressaltar que a vigilância da síndrome gripal e da síndrome respiratória aguda grave é feita através por meio da coleta de dados de centros sentinela e não inclui todos os casos da doença. Em estudo de coorte realizado no Peru, com investigação sorológica, foi possível verificar que, aproximadamente, $75 \%$ das infecções foram leves ou assintomáticas; $22 \%$ dos infectados apresentaram síndrome gripal, mas não procuram o médico e apenas 3\% buscaram atendimento médico. ${ }^{82} \mathrm{~A}$ análise desses resultados mostra que os dados de vigilância passiva subestimam a impacto da doença ${ }^{82,83}$.

Embora a maioria das infecções por vírus influenza tenha evolução autolimitada, a temperatura frequentemente é alta $38 \mathrm{C}$ a $40 \mathrm{C}$ e a febre dura 3 a 5 dias, enquanto a tosse e a fadiga podem se prolongar por duas semanas ou mais ${ }^{2}$.

Lactentes jovens infectados pelos vírus influenza podem apresentar quadro semelhante à sepse. Dor abdominal e diarreia são mais comuns em crianças e parecem ser mais frequentes após infecção pelos vírus B.12,91 Infecções de mucosas, como otite e sinusite, e o agravamento de crises de

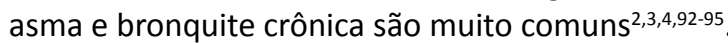

A pneumonia é a complicação da influenza que mais frequentemente leva à hospitalização e à morte; pode ser causada pelo próprio vírus ou por superinfecção bacteriana, principalmente, por Streptococcus pneumoniae ${ }^{96}$. A pneumonia por superinfecção bacteriana ocorre geralmente, uma semana após a infecção viral, fato que dificulta o diagnóstico da influenza, pois os vírus já não estão mais presentes nas secreções respiratórias ${ }^{39}$. Estudos anatomopatológicos em que foram utilizados testes histoquímicos e RT-PCR possibilitaram a identificação de vários casos de morte por influenza em crianças e adultos, demonstrando que o impacto da doença é muito maior do que o registrado em dados oficiais ${ }^{97}$.

Outras complicações como a exacerbação de doenças crônicas cardiovasculares, pulmonares e diabetes, infarto agudo do miocárdio, miocardite, pericardite, miosite, radomiólise, acidente vascular cerebral e manifestações neurológicas também podem ocorrer ${ }^{2,3}$.

A convulsão febril é a complicação neurológica mais comum em crianças menores de cinco anos, mas as cepas A e B também podem causar outras manifestações neurológicas graves, como encefalite. Existem evidências de que os vírus B estão mais associados ao desencadeamento de quadros neurológicos e musculares. ${ }^{12,20,98}$ Durante uma epidemia de influenza B registrada na Coreia em 2012, em um período de 5 meses, 7,9\% (28/355) dos pacientes pediátricos com diagnóstico confirmado de influenza apresentaram manifestações neurológicas ou musculares. A média de idade das crianças com essas manifestações foi de 48,7 meses, e os sintomas neurológicos/ musculares surgiram, em média, 2,2 dias após o início da síndrome gripal. A convulsão foi o problema mais comum, sendo registrada em $67,9 \%$ (19/28 casos), seguida por miosite $(17,9 \%)$, aumento na pressão intracraniana $(3,6 \%)$, delírio e cefaleia grave (3,6\%). Todas as convulsões foram febris e $15,8 \%$ eram convulsões complexas ${ }^{98}$.

De acordo com o CDC, as mortes causadas por infarto agudo do miocárdio, doença vascular e diabetes, não são contadas entre as mortes por Pneumonia e Influenza. Como a infecção viral prévia é fator de risco para essas mortes, o impacto da influenza certamente é subestimado ${ }^{3}$.

\section{Sazonalidade}

Os vírus influenza circulam durante todo o ano $0^{2,3,4,13}$. Nas regiões de clima temperado, observa-se aumento da circulação dos vírus influenza durante os meses mais frios, enquanto nas regiões de clima tropical a sazonalidade não é tão característica e a maior circulação dos vírus é observada durante as estações com maior índice de pluviosidade ${ }^{13,99}$. Em algumas temporadas, pode haver antecipação da circulação da influenza ou recrudescência da doença, quando surgem novas cepas de influenza $A$ ou $B^{27}$.

\section{Influenza na faixa etária pediátrica}

Crianças menores de cinco anos apresentam as mais elevadas taxas de infecção por influenza e são as principais transmissoras do vírus para seus contatos na família e na escola ${ }^{3}$. Estima-se que 10 a $30 \%$ das crianças sejam infectadas pelos vírus influenza durante as epidemias anuais e que, aproximadamente metade delas, necessite de atendimento médico ${ }^{76,85,90}$.

Durante o inverno, observa-se aumento de 30 a $50 \%$ nas consultas e 50 a $100 \%$ nos atendimentos em serviços de emergência e hospitalizações e muitas crianças são tratadas com antibióticos ${ }^{88}$.

Durante o período de maior circulação dos vírus ocorrem, em média, 6 a 15 consultas e são feitas 3 a 9 prescrições adicionais de antibióticos para cada 100 crianças $^{76}$. Estima-se que, para cada 1.000 crianças menores de cinco anos, anualmente, ocorram 50 a 95 consultas ambulatoriais e 6 a 27 consultas em serviços de emergência ${ }^{14}$ e que o número de consultas ambulatoriais seja em crianças de 24 a 59 meses seja quase 300 vezes superior à taxa de hospitalizações ${ }^{14}$.

Nos EUA, entre 2003 e 2010, a infecção por influenza foi confirmada em 7.293 crianças hospitalizadas; a análise de 6.769 casos revelou que, em média, a hospitalização durou três dias; 975 crianças (14\%) necessitaram de cuidados intensivos, 359 (5\%) tiveram insuficiência respiratória, e 40 (1\%) foram a óbito. 
A complicação mais comum foi a pneumonia (28\%) seguida por exacerbação de asma (22\%) em crianças maiores de dois anos e desidratação (21\%).28 As faltas dos pais ao trabalho para cuidar de crianças doentes foram estimadas entre 1,3 a 6,3 dias ${ }^{100}$.

As hospitalizações por influenza em crianças são inversamente proporcionais à idade, sendo mais elevadas em lactentes menores de seis meses $9,89,90,101-103$. Em crianças com idade entre 2 e 5 anos, a taxa de hospitalização por influenza é, pelo menos, quatro vezes superior à registrada em crianças maiores e adolescentes e tão elevada quanto a observada em idosos sem fatores de risco. Ao contrário dos adultos, a maioria das crianças que desenvolve complicações pós-influenza não apresenta qualquer doença de base $\mathrm{e}^{2,3,88}$.

Lactentes menores de seis meses de idade apresentam alto risco para hospitalização, mesmo quando não apresentam comorbidades $3,86,104-106$.

Em pessoas com mais de cinco anos, as complicações são mais comuns nos grupos que apresentam alguma doença de base, nas gestantes e em algumas populações que vivem em condições desfavoráveis, como indígenas e pessoas privadas de liberdade 2,3,100,107-110.

A infecção é muito comum em escolares, principalmente na escola elementar ${ }^{44,77,79,80,111,112}$. Em estudo realizado em Quebec, $35,6 \%$ de 3.432 escolas tiveram registro de absenteísmo acima de $10 \% \cdot{ }^{77}$.

Os dados da América Latina são muito mais limitados, entretanto uma meta-análise que incluiu 31 estudos publicados entre 1980 e 2008 verificou-se que a incidência de síndrome gripal foi 36.080 por 100.000 pessoas-ano e que o vírus influenza foi isolado em 4,6\% a 15,4\%; enquanto nas crianças menores de cinco anos a influenza foi confirmada em $25 \%$ dos casos, nas pessoas com mais de 14 anos, foi confirmada em $19 \%$. A maioria das cepas identificadas foi do tipo A ( $86 \%$ A e $14 \%$ B). A média de hospitalização nos casos positivos variou entre 5,8 e 12,9 dias, com custo por caso hospitalizado de US\$575

Na tabela 1, encontra-se a distribuição dos casos de influenza A e B identificados por meio da vigilância da síndrome gripal no Brasil entre 2002 e 2008, (antes da pandemia) ${ }^{17}$ e na tabela 2, a distribuição dos casos de influenza A e B identificados de 2010 a 2014 (período pós-pandêmico) ${ }^{13}$. Em ambas, pode-se observar grande variação na circulação das cepas A e B.

No Brasil, os vírus influenza são os principais agentes identificados em crianças com mais de dois anos idade com síndrome gripal $^{90}$ e frequentemente, existe cocirculação dos vírus influenza com outros vírus ${ }^{10,17,90}$. As cepas A e B podem circular concomitantemente ou em períodos diversos ${ }^{2,3,10,13,17,89}$. Globalmente, durante a epidemia causada pelo $A(H 1 N 1)$ pdm 09, tanto a incidência como as taxas de hospitalização aumentaram substancialmente em quase todas as faixas etárias, com exceção dos idosos. ${ }^{2,3,8,85,108,113,114}$ Interessante observar que, no Brasil, onde as coberturas vacinais em crianças de 6 a 24 meses e idosos com mais de 60 anos foram superiores a $80 \%$ no ano de 2013 , o maior número de casos hospitalizados e as maiores taxas de letalidade entre os casos com síndrome respiratória aguda grave foram registrados em adultos jovens ${ }^{14,22}$. Como a influenza não pode ser facilmente distinguida de outras infecções virais e bacterianas, é muito comum o aumento na prescrição de antibióticos ${ }^{16,13,32,81,115-117}$.

Tabela 1. Distribuição dos casos de influenza A e B identificados em casos de síndrome gripal no Brasil, de 2002 a 2008 (período pré-pandêmico) ${ }^{17}$.

\begin{tabular}{ccccc}
\hline & \multicolumn{2}{c}{ Influenza A } & \multicolumn{2}{c}{ Influenza B } \\
\cline { 2 - 5 } Ano & $\mathbf{N}$ & \% & $\mathbf{N}$ & $\%$ \\
\hline 2002 & 39 & 54,2 & 33 & 45,8 \\
2003 & 89 & 92,7 & 7 & 7,3 \\
2004 & 158 & 87,3 & 23 & 12,7 \\
2005 & 94 & 90,4 & 10 & 9,6 \\
2006 & 184 & 79,0 & 49 & 21,0 \\
2007 & 229 & 88,1 & 31 & 11,9 \\
2008 & 162 & 60,4 & 106 & 39,6 \\
\hline
\end{tabular}

2002-2008: $A=79 \%(955 / 1.214)$

2002-2008: $B=21 \%(259 / 1.214)$

Fonte: Freitas FT. Braz J Infect Dis. 2013

Tabela 2. Distribuição dos casos de influenza A e B identificados de 2010 a 2014 (período pós-pandêmico) ${ }^{13}$.

\begin{tabular}{rrrrrrrrrr}
\hline \multicolumn{10}{c}{ Brasil } \\
\hline Ano & $\begin{array}{c}\text { A(H1N1) } \\
\text { pdm09 }\end{array}$ & $\%$ & A(H3N2) & $\%$ & A? & $\%$ & B & $\%$ & Total \\
\hline 2010 & 641 & 60 & 202 & 19 & 82 & 7 & 150 & 14 & 1.075 \\
2011 & 253 & 25 & 593 & 59 & 3 & 1 & 156 & 15 & 1.005 \\
2012 & 722 & 45 & 684 & 43 & 44 & 3 & 139 & 9 & 1.589 \\
2013 & 2.802 & 60 & 482 & 10 & 0 & 0 & 1.415 & 30 & 4.699 \\
2014 & 29 & 30 & 65 & 66 & 0 & 0 & 4 & 4 & 98 \\
\hline
\end{tabular}

2010-2014: $A(\mathrm{H} 1 \mathrm{~N} 1)=52 \%(4.447 / 8.466)$

2010-2014: $A(H 3 N 2)=24 \%(2.026 / 8.466)$

2010-2014: $A$ ? $=2 \%(129 / 8.466)$

2010-2014: B=22\%(1.864/8.466)PAHO.

Distribution of sincitial respiratory vírus by region, 2010-2014 [Internet]. 2014 [cited 2014 June 2]. Available from: http://ais.paho.org/phip/viz/ed_flu.asp13

\section{Impacto econômico e social da influenza}

Nos EUA, estima-se que 5 a $20 \%$ da população adquiram influenza e mais de 200.000 pessoas sejam hospitalizadas todos os anos. O número anual de mortes entre 1976 e 2007 variou entre 3.000 e $49.000^{84}$. Embora a maioria das mortes seja registrada em adultos, crianças são mais frequentemente acometidas pela influenza, gerando enorme demanda aos serviços médicos em serviços ambulatoriais e de emergência, são mais frequentemente tratadas com antibióticos. De 2005/2006 a 2008/2009, a incidência de influenza nos EUA aumentou em todas as faixas etárias, com predomínio em crianças e adolescentes e, no mesmo período, os gastos com influenza aumentaram quatro vezes ${ }^{101}$.

O impacto econômico da influenza na América Latina também é considerado substancial ${ }^{15}$. No Brasil, embora o número de 
casos e mortes confirmadas pela influenza sejam menores do que os registrados nos EUA, desde a pandemia de 2009, podese constatar que a doença causa enorme impacto no país, particularmente nos estados do sul e sudeste s0,14,17,22,108. $^{1}$.

Todos os anos, durante as epidemias sazonais de influenza, aumentam os gastos diretos e indiretos associados às doenças respiratórias ${ }^{2,3,7,19,88,101,118-124}$.

O impacto econômico da doença está claramente associado à emergência de novas variantes dos vírus, particularmente quando essas variantes não estão incluídas nas vacinas. Durante a época de maior circulação, os vírus influenza são identificados em até $30 \%$ dos casos de síndrome gripal, ${ }^{3,10,13}$ principal motivo de consulta médica em serviços ambulatoriais, e a um aumento paralelo nas taxas de hospitalização por pneumonia ${ }^{2,3,28,76,88}$.

\section{Por que ampliar a vacinação contra influenza na faixa etária} pediátrica?

Estima-se que a ampliação da vacinação de crianças pode reduzir substancialmente a morbidade e a mortalidade em toda a população ${ }^{36,46}$. Embora o licenciamento de vacinas seja baseado em estudos que apenas evidenciam a, proteção direta, a proteção indireta oferecida pela vacinação contra influenza já foi demonstrada em diversos estudos tendo reduzido a mortalidade em idosos no Japão, ${ }^{125,126}$ o consumo de antibióticos em vacinados e não vacinados ${ }^{111}$, assim como o absenteísmo de alunos e professores ${ }^{80,127}$.

Durante o período de maior circulação da influenza, observase aumento no absenteísmo à escola. Esse problema tem sido amplamente estudado no Japão, onde uma taxa de absenteísmo de $10 \%$ dos alunos leva ao fechamento da escola ou da classe onde os casos são registrados. O fechamento de escolas parece ter potencial para reduzir a transmissão da influenza, mas essa estratégia tem grande repercussão para a criança e a família. ${ }^{40}$ Embora as medidas não farmacêuticas, como o uso de máscaras e higiene, e o uso de antivirais, possam reduzir a transmissão da influenza, a efetividade desses recursos para controlar a disseminação dos vírus é variável e existem enormes dificuldades para sua implementação em tempo hábil, considerando-se que a excreção dos vírus tem início antes do aparecimento dos sintomas e que as crianças pequenas ainda não têm maturidade suficiente para seguir rigorosamente as normas de higiene ${ }^{128-133}$.

A ampliação da vacinação na faixa etária pediátrica também pode gerar economia, principalmente se forem considerados os custos decorrentes do absenteísmo dos pais ao trabalho e a proteção indireta ${ }^{117,121}$.

Nos EUA, o impacto da vacinação contra a influenza na carga da doença e hospitalizações revelou que, em média, a vacinação reduziu 1,1 milhão de casos entre 2006 e 2011, evitou entre 7.700 a 40.400 hospitalizações de acordo com a temporada estudada. O benefício da vacinação variou com faixa etária e aumentou proporcionalmente de acordo com o aumento das coberturas vacinais. Na faixa etária de 0 a 4 anos, entre 2005/2006 e 2010 /2011, a fração evitada de casos de influenza aumentou de aproximadamente $12 \%$ para mais de $28 \%$. Nesse estudo não foi avaliado o impacto resultante da proteção indireta, mas os autores concluem que o aumento nas coberturas vacinais em não idosos deve ter contribuído para a efetividade do programa ${ }^{119}$.

Além disso, em uma pesquisa que incluiu 24.195 famílias, 18,9\% de um total 8.976 adultos portadores de condição de risco para complicações de influenza tinham contato domiciliar com crianças. Embora a vacina fosse recomendada para contatos domiciliares de pessoas com alto risco para complicações, as coberturas vacinais eram de apenas $30 \%$ em $2006 .{ }^{134} \mathrm{~A}$ ampliação da vacinação certamente contribuirá para proteger indiretamente os grupos de risco ${ }^{3,8}$.

Em estudo prospectivo com monitoramento durante 12 semanas, o absenteísmo entre estudantes não vacinados (39\%) foi significativamente maior em comparação com o grupo vacinado $(27 \%)^{126}$. Entre 2009 e 2010, a vacinação de escolares foi capaz de reduzir o absenteísmo entre os estudantes vacinados e seus professores não vacinados. Embora o benefício indireto da vacinação tenha sido considerado modesto, com redução de $8 \%$ na incidência de síndrome gripal em ambos os grupos, a vacinação foi realizada no auge da circulação dos vírus, o que poderia ter reduzido seu impacto ${ }^{44}$. Quando se avalia o impacto da vacinação em percentual de casos, não se tem uma ideia precisa do benefício em termos de número de casos, hospitalizações e mortes evitadas. Em recente estudo realizado pelo $C D C$, verificou-se que, de agosto a dezembro de 2009, foram registrados nos EUA mais de 800 interrupções nas atividades letivas, envolvendo uma ou mais escolas. $O$ impacto dessas interrupções afetou 1.942 escolas, 623.616 estudantes e 40.521 professores $^{133}$.

As vacinas contra influenza são seguras e efetivas, embora a proteção varie de acordo com faixa etária e imunidade prévia (natural ou adquirida por vacinação) 2,3,8,109.

Embora os lactentes menores de seis meses de idade não possam receber a vacina influenza, essas crianças podem se beneficiar da vacinação de gestantes e puérperas. Desde 2012, a OMS coloca as gestantes como grupo prioritário para imunização em todos os países devido aos benefícios à mãe e ao recém-nascido ${ }^{109}$.

Os outros grupos de risco, como crianças de 6 a 60 meses, idosos, portadores de co-morbidades, profissionais de saúde, não estão listados em ordem de preferência; entretanto, estima-se que a vacinação de crianças, particularmente os pré-escolares, terá maior impacto na coletividade ${ }^{47,134}$ devido à redução na transmissão dos vírus e na proteção indireta dos não vacinados. Além disso, grande percentual de crianças convive com grupos de risco, como irmãos menores de seis meses, gestantes e portadores de comorbidades ${ }^{135}$.

\section{Vacinas trivalentes e vacinas tetravalentes:}

Existem diversas vacinas licenciadas para uso em crianças, 
contendo antígenos dos vírus $\mathrm{A}$ e $\mathrm{B}$, com ou sem adjuvantes, e vacinas contendo vírus vivos atenuados ${ }^{2,3,4}$. A maioria das vacinas licenciadas para combater a influenza é trivalente, sendo composta por antígenos de duas cepas da influenza A e uma B $4,8,136$ e, desde 2012 , existem recomendações para uso de novas vacinas tetravalentes, contendo antígenos de duas cepas $A$ e duas $B$, já licenciadas em alguns países ${ }^{4,8,137}$. Nos últimos 10 anos, em aproximadamente metade das temporadas de influenza nos EUA, Europa, Austrália e Brasil, a cepa B incluída nas vacinas trivalentes não era compatível com as cepas circulantes .21,138-140. $^{2}$. A falta de proteção cruzada contra a linhagem não incluída na vacina pode comprometer a efetividade da vacina ${ }^{12,21,24,138}$, por esse motivo, estima-se que, em breve, as vacinas tetravalentes substituirão as trivalentes. Estudos de custo-benefício indicam que a substituição de vacinas trivalentes por vacinas tetravalentes trará benefícios econômicos, além de reduzir o impacto da doença na comunidade ${ }^{19}$. No Brasil, espera-se que as novas vacinas tetravalentes sejam licenciadas em breve. 0 aprimoramento da vigilância epidemiológica, com introdução de métodos mais sensíveis para o diagnóstico da doença, a avaliação do impacto das diferentes cepas em cada faixa etária e em estudos farmacoeconômicos serão essenciais para avaliar os benefícios dessas novas vacinas nos próximos anos.

\section{CONCLUSÃO}

Os estudos recentemente publicados indicam que a influenza causa substancial impacto na infância e que as crianças são as principais transmissoras dos vírus na comunidade. A vacinação é a melhor forma de prevenir a infecção por influenza e suas complicações em crianças e adultos e a ampliação da vacinação de crianças pode reduzir substancialmente a morbidade e a mortalidade em toda a população e gerar economia para a família e a sociedade.

\section{REFERÊNCIAS}

1.WHO. Media Centre. Influenza (seasonal). Fact sheet no 211, Mar 2014 [Internet]. 2014 [cited 2014 May 29]. Available from: http://www.who.int/ mediacentre/factsheets/fs211/en/index.html

2.Fiore $A E$, Bridges $C B$, Katz JM, Cox NJ. Inactivated influenza vaccines. In: Plotkin S, Orenstein W, Offit P, editors. Vaccines. Philadelphia. Saunders Elsevier; 2013. P. 257-93.

3.Centers for Disease Control and Prevention (CDC). Prevention and control of seasonal influenza with vaccines: recommendations of the Advisory Committee on Immunization Practices - United States, 2013-2014. MMWR Recomm Rep. 2013 Sept 20; 62 (RR07):1-43. PubMed PMID: 24048214. Errata em: MMWR. 2013 Nov 15, 62(45): 906.

4.WHO. Recommended composition of influenza virus vaccines for use in the 2014 southern hemisphere influenza season. Wkly Epidemiol Rec. 2013 Oct 11;88(41):437-48.

tion\%3Dcom_docman\%26task\% RHMjksASzkoLYBg\&usg=A

5.Pan American Health Organization. Technical Advisory Group on Vaccinepreventable Diseases XXI Meeting: Vaccination: a shared responsibility [Internet]; 2013 July 3-5; Quito, Ecuador. [citado 2014 mês dia] Available from: http://www.google.com.br/l?sa=t\&rct=j\&q=\&esrc=s\&frm=1\&source=web\&c $\mathrm{d}=5$ \&ved=0CFEQFjAE\&url=http\%3A\%2F\%2Fwww. paho.org\%2Fhq\%2Findex. php\%3Foption\%3Dcom docman\%26task\%3Ddoc download\%26gid\%3D2242 3\%26Itemid\%3D270\&ei=H9oEU9SRHMjksASzkoLYBg\&usg=AFQjCNG1m0cHyx Okduu9wSq8VqMehPsjMg\&sig2=uSaCSILs63-NiR5gC2STbw\&bvm=bv.61535280, d.dmQ\&cad=rja

6.WHO. Vaccine-preventable diseases: monitoring system, 2014 global summary. Immunization schedule selection centre [Internet]. 2014 [updated 2014 Mar 13; cited 2014 Mar 13]. Available from: http://apps.who.int/ immunization_monitoring/globalsummary/s?schedulecriteria\%5Bdummy\%5D $=\&$ schedulecriteria $\% 5$ Bvaccine $\% 5 D \% 5 B \% 5 D=D T I P V \&$ schedulecriteria $\% 5 B O K \%$ $5 \mathrm{D}=\mathrm{OK}$

7.Fiore AE, Shay DK, Haber P, Iskander JK, Uyeki TM, Mootrey G, et al. Prevention and control of influenza. Recommendations of the Advisory Committee on Immunization Practices (ACIP), 2007. MMWR Recomm Rep. 2007 July 13; 56(RR-6):1-54. PubMed PMID: 17625497.

8.Committee on infectious diseases. Recommendations for prevention and control of influenza in children, 2013-2014. Pediatrics. 2013 Oct 4; 132(4):e1089104. doi: $10.1542 /$ peds.2013-2377.

9.Heikkinen T, Silvennoinen H, Reunanen T, Ziegler T, Vainionpää R. Illness symptoms and absences due to influenza in different age groups of children. International Congress Series. 2004 June; 1263:101-4. doi: 10.1016/j. ics.2004.04018.

10.Ministério da Saúde (Brasil), Coordenação Geral do Programa Nacional de Imunizações. Informe Técnico: Campanha nacional de vacinação contra a influenza. [Internet]. Brasília: Ministério da Saúde; 2014 [citado 2014 abr 28]. Disponívelem:http://sprs.com.br/sprs2013/bancoimg/140402011250Informe_ Campanha_Influenza_25_03_2014.pdf

11.Samaan G, McPherson M, Partridge J. A review of the evidence to support influenza vaccine introduction in countries and areas of WHO's Western Pacific Region. Plos ONE. 2013 July 16;8(7):e70003. doi: 10.1371/journal. pone0070003.

12.Paul Glezen W, Schmier JK, Kuehn CM, Ryan KJ, Oxford J. The burden of influenza B: a structured literature review. Am J Public Health. 2013 Mar; 103(3):e43-51. doi: 10.2105/APH.2012.301137. PubMd PMID: 3327249.

13.Pan American Health Organzation . Influenza and other respiratory viruses under surveillance, 2010-2014 [Internet]. 2014 [updated 2014 Mar 12; cited 2014 Mar 12]. Available from: http://ais.paho.org/phip/viz/ed_flu.asp

14.Bricks L, Moraes JC. Influenza B impact in pediatric age groups: analysis of 5,883 confirmed influenza cases in SARS inpatients, Brazil 2013 [poster on the Internet]. In: 32 ㅇ Annual Meeting of the European Society for Paediatric Infectious Diseases; 2014 May 6-10; Dublin, Ireland [cited 2014 May 12]. Available from: http://espid.meetingxpert.net/espid_945/poster_95421/ program.aspx

15.Savy V, Ciapponi A, Bardach A, Glujovsky D, Aruj P, Mazzoni A, et al. Burden of influenza in Latin America and the Caribbean: a systematic review and metaanalysis. Influenza Other Respi Viruses. 2013 Nov;7(6):1017-32. doi: 10.1111/ irv.12036. PubMed PMID: 23210504

16.Wang D, Zhang T, Wu J, Jiang $Y$, Ding $Y$, Hua J, et al. Socio-economic burden of influenza among children younger than 5 years in the outpatient setting in Suzhou, China. Plos ONE. 2013 ago 8. 8(8):e69035. doi: 10.1371/journal. pone0069035.

17.Freitas FT. Sentinel surveillance of influenza and other respiratory viruses, Brazil, 2000-2010. Braz J Infect Dis. 2013 Jan-Feb; 17(1):62-8. doi: 10.1016j. bjid.2012.09.001.

18. Mook P, Ellis J, Watson JM, Thompson Cl, Zambon M, McMenamin J, et al. Public health implications of influenza $B$ outbreaks in closed settings in the United Kingdom in the 2007/08 influenza season. Euro Surveill. 2008 Sept 
18;13(38). pii: 18986.

19.Karve S, Meier G, Davis KL, Misurski DA, Wang CC. Influenza-related health care utilization and productivity losses during seasons with and without a match between the seasonal and vaccine virus B lineage. Vaccine. 2013 July 18; 31(33):3370-88.

20. Reed C, Meltzer MI, Finelli L, Fiore A. Public health impact of including two lineages of influenza $B$ in a quadrivalent seasonal influenza vaccine. Vaccine. 2012 Mar 2; 30(11):1993-8. doi: 10.1016/jvaccine.2011.12.098. PubMed PMID: 2222686 .

21. Ambrose CS, Levin MJ. The rationale for quadrivalent influenza vaccines. Hum Vaccin Immunother. 2012 Jan; 8(1):81-8. doi: 10.4161/hv.8.1.17623. PubMed PMID: 22252006.

22. Bricks L, Carvalhanas TRMP, Ribeiro AF, Ferreira PM, Paiva TM, Yu ALF, et al. Analysis of 2,748 confirmed influenza cases in serious acute respiratory syndrome (sars) hospitalizations in São Paulo state (SP), Brazil - 2013 In: 320 Annual Meeting of the European Society for Paediatric Infectious Diseases; 2014 May 6-10; Dublin, Ireland [cited 2014 May 12]. Available from: http:// espid.meetingxpert.net/ESPID_945/poster_94756/program.aspx/anchor94756

23. Olson DR, Heffernan RT, Paladini M, Konty K, Weiss D, Mostashari F Monitoring the impact of influenza by age: emergency department fever and respiratory complaint surveillance in New York City. PloS Med. 2007 Aug 7, 4(8):e247. doi:10.1371/journal.pmed.0040247

24. Tricco AC, Chit A, Soobiah C, Hallett D, Meier G, Chen MH, et al. Comparing influenza vaccine efficacy against mismatched and matched strains: a systematic review and meta-analysis. BMC Med. 2013 June 25;11:153. doi:10.1186/17417015-11-153

25. Janjua NZ, Skowronski DM, De Serres G, Dickinson J, Crowcroft NS, Taylor M, et al. Estimates of influenza vaccine effectiveness for 2007-2008 from Canada's sentinel surveillance system: cross-protection against major and minor variants. J Infect Dis. 2012 June

15; 205(12):1858-68. doi: 10.1093/infdis/jis283. PubMed PMID: 22492921

26. Neuzil KM, Mellen BG, Wright PF, Mitchel Jr EF, Griffin MR. The effect of influenza on hospitalizations, outpatient visits, and courses of antibiotics in children. N Engl J Med. 2000 Jan 27; 342(4):225-31. doi: 10.1056/ NEJM200001273420401

27. CDC. Fluview: a weekly influenza surveillance report prepared by the Influenza Division [Internet]. 2014 [cited 2014 may 20]. Available from: http:// www.cdc.gov/flu/weekly/

28. Dawood FS, Chaves SS, Pérez A, Reingold A, Meek J, Farley MM, et al. Complications and associated bacterial coinfections among children hospitalized with seasonal or pandemic influenza, United States, 2003-2010. J Infect Dis. 2014 Mar 1;209(5):686-94. doi: 10.1093/infdis/jit473. PubMed PMID: 23986545.

29. Glatman-Freedman A, Portelli I, Jacobs SK, Mathew JI, Slutzman JE, Goldfrank $L R$, et al. Attack rates assessment of the 2009 pandemic H1N1 influenza $A$ in children and their contacts: a systematic review and meta-analysis. Plos ONE. 2012 Nov 30; 7(11):e50228. doi: 10.1371/journal.pone.0050228

30. Jain S, Kamimoto L, Bramley AM, Schmitz AM, Benoit SR, Louie J, et al. Hospitalized patients with 2009 H1N1 influenza in the United States, AprilJune 2009. N Engl J Med. 2009 Nov 12;361(20):1935-44. doi: 10.1056/ NEJMoa0906695

31. O'Riordan S, Barton M, Yau Y, Read SE, Allen U, Tran D. Risk factors and outcomes among children admitted to hospital with pandemic H1N1 influenza. CMAJ. 2010 Jan 12; 182(1):39-44. doi: 10.1503/cmaj.091724. Pubmed PMID: 19926677

32. Cox CM, D'Mello T, Perez A, Reingold A, Gershman K, Yousey-Hindes K, et al. Increase in rates of hospitalization due to laboratory-confirmed influenza among children and adults during the 2009-10 influenza pandemic. J Infect Dis. 2012 Nov; 206(9):1350-8. doi: 10.1093/infdis/jis517. PubMEd PMID: 23002448.
33. Thompson WW, Moore MR, Weintraub E, Cheng PY, Jin X, Bridges CB, et al. Estimating influenza-associated deaths in the United States. Am J Public Health. 2009 Oct; 99 Suppl 2:S225-30. doi: 10.2105/AJPH.2008.151944. PubMed PMID: 19797736

34. Secretaria de Salud (Mexico). Casos y defunciones confirmadas a influenza por entidad federativa e institución: México 2014. [Internet]. Mexico: Secretaira de salud; 2014. [actualizado 2014 Mar 6; citado em 2014 Mar 14]. Disponible en: http://www.epidemiologia.salud.gob.mx/doctos/lineamientos/influenza/ corte060314.pdf

35. Flannery B, Thaker SN, Clippard J, Monto AS, Ohmit SE, Zimmerman RK, et al. Interim estimates of 2013-14 seasonal influenza vaccine effectiveness - United States, february 2014. MMWR Morb mortal wkly rep. 2014 Feb 21; 63(7):137-42.

36. Plans-Rubió $P$. The vaccination coverage required to establish herd immunity against influenza viruses. Prev Med. 2012 July; 55(1):72-7. doi: 10.1016/j. ypmed.2012.02.015. PubMed PMID: 22414740

37. Bischoff WE, Swett K, Leng I, Peters TR. Exposure to influenza virus aerosols during routine patient care. J Infect Dis. 2013 Apr;207(7):1037-46. doi: 10.1093/ infdis/jis773. PubMed PMID: 23372182.

38. Milton DK, Fabian MP, Cowling BJ, Grantham ML, McDevitt JJ. Influenza virus aerosols in human exhaled breath: particle size, culturability, and effect of surgical masks. PLoS Pathog. 2013 Mar;9(3):e1003205. doi:10.1371/journal. ppat.1003205

39. Suess T, Remschmidt C, Schink SB, Schweiger B, Heider A, Milde J, et al. Comparison of shedding characteristics of seasonal influenza virus (sub) types and influenza A(H1N1)pdm09; Germany, 2007-2011. Plos ONE. 2012; 7(12):e51653. doi:10.1371/journal.pone.0051653.

40. Jackson C, Vynnycky E, Hawker J, Olowokure B, Mangtani P. School closures and influenza: systematic review of epidemiological studies. BMJ Open. 2013 Feb 26;3(2). pii: e002149. doi: 10.1136/bmjopen-2012-002149. PubMed PMID: 23447463

41. Reichert TA, Simonsen L, Sharma A, Pardo SA, Fedson DS, Miller MA. Influenza and the winter increase in mortality in the United States, 1959-1999. Am J Epidemiol. 2004 Sept 1;160(5):492-502. PubMed PMID: 15321847.

42. Nukiwa-Souma N, Burmaa A, Kamigaki T, Od I, Bayasgalan N, Darmaa B, et al. Influenza transmission in a community during a seasonal influenza $A(H 3 N 2)$ outbreak (2010-2011) in Mongolia: a community-based prospective cohort study. Plos ONE. 2012 Mar 13;7(3):e33046. doi: 10.1371/journal.pone.0033046

43. Petrie JG, Ohmit SE, Cowling BJ, Johnson E, Cross RT, Malosh RE, et al. Influenza transmission in a cohort of households with children: 2010-2011. Plos ONE. 2013 Sep 25; 8(9):e75339. doi: 10.1371/journal.pone.0075339.

44. Graitcer SB, Dube NL, Basurto-Davila R, Smith PF, Ferdinands J, Thompson $M$, et al. Effects of immunizing school children with 2009 influenza $A(H 1 N 1)$ monovalent vaccine on absenteeism among students and teachers in Maine. Vaccine. 2012 July 6;30(32):4835-41. doi: 10.1016/j.vaccine.2012.05.008. PubMed PMID: 22609012.

45. Kim JH, Lee DH, Shin SS, Kang C, Kim JS, Jun BY, et al. In-flight transmission of novel influenza A (H1N1). Epidemiol Health. 2010 maio 31; 32:e2010006. doi: 10.4178/epih/e2010006.

46. Pitman RJ, White LJ, Sculpher M. Estimating the clinical impact of introducing paediatric influenza vaccination in England and Wales. Vaccine. 2012 Feb; 30(6):1208-24. doi: 10.1016/j.vaccine.2011.11.106. PubMed PMID: 22178725

47. Shim E. Optimal strategies of social distancing and vaccination against seasonal influenza. Math Biosci Eng. 2013 Out-Dez;10(5-6):1615-34. doi: 10.3934/mbe.2013.10.1615.

48. Seale H, Weston KM, Dwyer DE, Zhu M, Allchin L, Booy R, et al. The use of oseltamivir during an influenza $B$ outbreak in a chronic care hospital. Influenza Other Respir Viruses. 2009 Jan; 3(1):15-20. doi: 10.1111/j.1750- 


\subsubsection{3.x. PubMed PMID 19453437}

49. Van Voris LP, Belshe RB, Shaffer JL. Nosocomial influenza B virus infection in the elderly. Ann Intern Med. 1982 Feb; 96(2):153-8. doi:10.7326/0003-481996-2-153.

50. Meibalane R, Sedmak GV, Sasidharan P, Garg P, Grausz JP. Outbreak of influenza in a neonatal intensive care unit. J Pediatr. 1977 dez; 91(6):974-6. PubMed PMID:

51. Liu PY, Wang LC, Lin YH, Tsai CA, Shi ZY. Outbreak of influenza A and B among military recruits: evidence from viral culture and polymerase chain reaction. J Microbiol Immunol Infect. 2009 Apr;42(2):114-21. PubMed PMID: 19597642

52. Pyhälä R, Visakorpi R, Ikonen N, Kleemola M. Influence of antigenic drift on the intensity of influenza outbreaks: upper respiratory tract infections of military conscripts in Finland. J Med Virol. 2004 Feb;72(2):275-80. PubMed PMID: 14695670

53. Saldarriaga T, Laguna-Torres VA, Arrasco J, Guillén L, Águila J, Águilar $P$, et al. Características clínicas y moleculares de un brote de influenza en dos bases militares, Tumbes- Perú, 2007. Rev Peru med exp salud publica. 2008 JanMar;25(1):35-43

54. Johnston F, Krause V, Miller N, Barclay L. An outbreak of influenza B among workers on an oil rig. Commun Dis Intell. 1997 Apr 17;21(8):106. PubMed PMID: 9140102.

55. Tohma K, Suzuki A, Otani K, Okamoto $M$, Nukiwa $N$, Kamigaki T, et al. Monitoring of influenza viruses in the aftermath of the Great East Japan earthquake. Jpn J Infect Dis. 2012; 65(6):542-4. PubMed PMID: 2183209.

56. Ferson $M$, Paraskevopoulos $P$, Hatzi $S$, Yankos $P$, Fennell $M$, Condylios $A$ Presumptive summer influenza $A$ : an outbreak on a trans-Tasman cruise. Commun Dis Intell. 2000 Mar 16; 24(3):45-7. PubMed PMID: 10812749.

57. Tarabbo M, Lapa D, Castilletti C, Tommaselli P, Guarducci R, Lucà $G$, et al. Retrospective investigation of an influenza $A / H 1 N 1 p d m$ outbreak in an Italian military ship cruising in the Mediterranean Sea, May-September 2009. Plos ONE. 2011 Jan 20;6(1):e15933. doi: 10.1371/journal.pone.0015933. PubMed PMID: 21283749.

58. Brotherton JM, Delpech VC, Gilbert GL, Hatzi S, Paraskevopoulos PD, McAnulty $J M$, et al. A large outbreak of influenza $A$ and $B$ on a cruise ship causing widespread morbidity. Epidemiol Infect. 2003 Apr;130(2):263-71. PubMed PMID: 12729195.

59. Miller JM, Tam TW, Maloney S, Fukuda K, Cox N, Hockin J, et al. Cruise ships: high-risk passengers and the global spread of new influenza viruses. Clin Infect Dis. 2000 Ago; 31(2):433-8. PubMed PMID: 10987701.

60. Uyeki TM, Zane SB, Bodnar UR, Fielding KL, Buxton JA, Miller JM, et al Large summertime influenza $A$ outbreak among tourists in Alaska and the Yukon Territory. Clin Infect Dis. 2003 Maio 1; 36(9):1095-102. PubMed PMID: 12715302.

61.Centers for Disease Control and Prevention . Influenza B virus outbreak on a cruise ship--Northern Europe, 2000. MMWR Morb Mortal Wkly Rep. 2001 Mar 2;50(8):137-40. PubMed PMID: 11393483.

62. Foxwell $A R$, Roberts $L$, Lokuge $K$, Kelly PM. Transmission of influenza on international flights, may 2009. Emerg Infect Dis. 2011 JulY;17(7):1188-94.

63. Baker MG, Thornley CN, Mills C, Roberts S, Perera S, Peters J, et al. Transmission of pandemic A/H1N1 2009 influenza on passenger aircraft: retrospective cohort study. BMJ. 2010 May 21;340:c2424. doi: 10.1136/bmj. c2424. PubMed PMID: 2095017.

64. Botelho-Nevers E, Gautret P. Outbreaks associated to large open air festivals, including music festivals, 1980 to 2012. Euro Surveill. 2013 Mar 14;18(11):20426. PubMed PMID: 23517872.

65. Blyth CC, Foo H, Van Hal SJ, Hurt AC, Barr IG, McPhie K, et al. Influenza outbreaks during World Youth Day 2008 mass gathering. Emerg Infect Dis. 2010 May;16(5):809-15. doi: 10.3201/eid1605.091136. PubMed PMID: 20409371.
66. Van Hal SJ, Foo H, Blyth CC, McPhie K, Armstrong P, Sintchenko V, et al. Influenza outbreak during Sydney World Youth Day 2008: the utility of laboratory testing and case definitions onmass gathering outbreak containment Plos ONE. 2009 Sep 3;4(9):e6620. doi: 10.1371/journal.pone.0006620. PubMed PMID: 19727401.

67. Lim HC, Cutter J, Lim WK, Ee A, Wong YC, Tay BK. The influenza A (H1N12009) experience at the inaugural Asian Youth Games Singapore 2009: mass gathering during a developing pandemic. Br J Sports Med. 2010 June; 44(7):52832. doi: 10.1136/bjsm.2009.069831. PubMed PMID: 20519257.

68. Dublineau A, Batéjat $C$, Pinon A, Burguière AM, Leclercq I, Manuguerra JC. Persistence of the 2009 pandemic influenza $A$ (H1N1) virus in water and on non-porous surface. PloS One. 2011; 6(11):e28043. doi: 10.1371/journal. pone.0028043. Epub 2011 Nov 23. PubMed PMID: 22132205.

69. Amorim AR, Fornells LA, Reis FC, Rezende DJ, Mendes GS, Couceiro JN, et al. Influenza A virus infection of healthy piglets in an abattoir in Brazil: animalhuman interface and risk for interspecies transmission. Mem Inst Oswaldo Cruz. 2013 Aug; 108(5):548-53. PubMed PMID: 23903968.

70. WHO. Influenza at the human-animal interface: summary and assessment as of 7 October 2013 [Internet]. 2013 [updated 2013 Oct 7; cited 2013 Oct 15]. Available from: http://www.who.int/influenza/human_animal_interface/ Influenza_Summary_IRA_HA_interface_7October13.pdf

71. Centers for Disease Control and Prevention. Seasonal Influenza (Flu): influenza A (H3N2) variant virus [Internet]. 2012 [updated 2012 Oct 29; cited 2014 Mar 18]. Available from: http://www.cdc.gov/flu/swineflu/h3n2v-cases. htm

72. WHO. Cumulative number of confirmed human cases for avian influenza A(H5N1) reported to WHO, 2003-2013 [Internet]. 2013 [cited 2013 Oct 15]. Available from: http://www.who.int/influenza/human_animal_interface/EN_ GIP_20131008CumulativeNumberH5N1cases.pdf

73. WHO. Global Alert and Response (GAR). Human infection with avian influenza A(H7N9) virus - update [Internet]. 2014 [updated 2014 Mar 17; cited 2014 Mar 18]. Available from: http://www.who.int/csr/don/2014_03_17_ h7n9/en/

74. Monto AS, Dickson CB, Landis JR. Utilization and acceptability of influenza $A /$ New Jersey/76 virus vaccine in Oakland County, Michigan. J Infect Dis. 1977 Dec;136 Suppl:S693-8. PubMed PMID: 606793

75.Glezen WP, Couch RB. Interpandemic influenza in the Houston area, 197476. N Engl J Med. 1978 Mar 16;298(11):587-92. PubMed PMID 628375.

76. Neuzil KM, Zhu Y, Griffin MR, Edwards KM, Thompson JM, Tollefson SJ, et al. Burden of interpandemic influenza in children younger than 5 years: 25 year prospective study. J Infect Dis. 2002 Jan 15;185(2):147-52. PubMed PMID 11807687.

77. Kom Mogto CA, De Serres G, Douville Fradet M, Lebel G, Toutant S, Gilca $\mathrm{R}$, et al. School absenteeism as an adjunct surveillance indicator: experience during the second wave of the 2009 H1N1 pandemic in Quebec, Canada. PloS One. 2012;7(3):e34084. doi: 10.1371/journal.pone.0034084. PubMEd PMID: 22479531.

78. Heikkinen T, Tsolia M, Finn A. Vaccination of healthy children against seasonal influenza: a European perspective. Pediatr Infect Dis J. 2013 Aug;32(8):881-8. doi: 10.1097/INF.0b013e3182918168. PubMed PMID: 23856713.

79. Barclay VC, Smieszek T, He J, Cao G, Rainey JJ, Gao H, et al. Positive network assortativity of influenza vaccination at a high school: implications for outbreak risk and herd immunity. Plos ONE. 2014 Feb 5;9(2):e87042. doi: 10.1371/ journal.pone.0087042. eCollection 2014. PubMed PMID: 24505274.

80. Hull HF, Ambrose CS. The impact of school-located influenza vaccination programs on student absenteeism: a review of the U.S. literature. J Sch Nurs. 2011 Feb;27(1):34-42. doi: 10.1177/1059840510389182. PubMed PMID: 21078842

81. Nichol KL, D’Heilly SJ, Ehlinger EP. Influenza vaccination among college and 
university students: impact on influenzalike illness, health care use, and impaired school performance. Arch Pediatr Adolesc Med. 2008 Dec;162(12):1113-81. doi: 10.1001/archpedi.162.12.1113. PubMed: 19047537.

82. Razuri H, Romero C, Tinoco Y, Guezala MC, Ortiz E, Silva M, et al. Populationbased active surveillance cohort studies for influenza: lessons from Peru. Bull World Health Organ. 2012 Apr 1;90(4):318-20. doi: 10.2471/BLT.11.097808. PubMed PMID: 22511830.

83. Hayward AC, Fragaszy EB, Bermingham A, Wang L, Copas A, Edmunds WJ, et al. Comparative community burden and severity of seasonal and pandemic influenza: results of the Flu Watch cohort study. Lancet Respir Med. 2014 June; 2(6): 445-54. doi: 10.1016/S2213-2600(14)70034-7. PubMed PMID: 24717637.

84. Centers for Disease Control and Prevention . Estimated influenza illnesses and hospitalizations averted by influenza vaccination - United States, 2012-13 influenza season. MMWR Morb Mortal Wkly Rep. 2013 Dec 13;62(49):9971000. PubMed PMID: 24336131

85. Griffin MR. Influenza vaccination: a 21st century dilemma. S D Med. 2013; Spec no:110-8. PubMed PMID: 23444601.

86. Neuzil KM, Hohlbein C, Zhu Y. Illness among schoolchildren during influenza season: effect on school absenteeism, parental absenteeism from work, and secondary illness in families. Arch Pediatr Adolesc Med. 2002 Oct;156(10):986 91. PubMed PMID: 12361443

87. Poehling KA, Edwards KM, Weinberg GA, Szilagyi P, Staat MA, Iwane MK, et al. The underrecognized burden of influenza in young children. N Engl J Med. 2006 July 6;355(1):31-40. PubMed PMID: 16822994

88. Menec VH, Black C, MacWilliam L, Aoki FY. The impact of influenzaassociated respiratory illnesses on hospitalizations, physician visits, emergency room visits, and mortality. Can J Public Health. 2003 Jan-Feb;94(1):59-63. PubMed PMID: 12583681.

89. Moore DL, Vaudry W, Scheifele DW, Halperin SA, Déry P, Ford-Jones E, et al. Surveillance for influenza admissions among children hospitalized in Canadian immunization monitoring program active centers, 2003-2004. Pediatrics. 2006 Sep;118(3):e610-9. PubMed PMID: 16950953.

90. Bricks LF. Crianças saudáveis devem receber a vacina contra influenza? Pediatria (São Paulo) 2004;26:49-58.

91.Nitsch-Osuch A, Wozniak-Kosek A, Korzeniewski K, Zycinska K, Wardyn K, Brydak LB. Clinical features and outcomes of influenza A and B infections in children. Adv Exp Med Biol. 2013;788:89-96. doi: 10.1007/978-94-007-66273_14. PubMed PMID: 23835964.

92.Kloepfer KM, Olenec JP, Lee WM, Liu G, Vrtis RF, Roberg KA, et al. Increased H1N1 infection rate in children with asthma. Am J Respir Crit Care Med. 2012 June 15;185(12):1275-9. doi: 10.1164/rccm.201109-16350C. PubMed PMID: 22366048

93. Miller JM, Tam TW, Maloney S, Fukuda K, Cox N, Hockin J, et al. Cruise ships: high-risk passengers and the global spread of new influenza viruses. Clin Infect Dis. 2000 Aug;31(2):433-8. PMID: 10987701.

94. Obuchi M, Adachi Y, Takizawa T, Sata T. Influenza A(H1N1)pdm09 virus and asthma. Front Microbiol. 2013 Oct 14;4:307. doi: 10.3389/fmicb.2013.00307. PubMed PMID: 24133489.

95. Oliveira PD, Menezes AM, Bertoldi AD, Wehrmeister FC. Inhaler use in adolescents and adults with self-reported physician-diagnosed asthma, bronchitis, or emphysema in the city of Pelotas, Brazil. J Bras Pneumol. 2013 May-June39(3):287-95. doi: 10.1590/S1806-37132013000300005. PubMed PMID: 23857689.

96. Klugman KP, Chien YW, Madhi SA. Pneumococcal pneumonia and influenza: a deadly combination. Vaccine. 2009 Aug 21;27 Suppl 3:C9-C14. doi: 10.1016/j. vaccine.2009.06.007. PubMed PMID: 19683658.

97. do Carmo Debur M, Raboni SM, Flizikowski FB, Chong DC, Persicote AP, Nogueira MB, et al. Immunohistochemical assessment of respiratory viruses in necropsy samples from lethal non-pandemic seasonal respiratory infections. J Clin Pathol. 2010 Oct;63(10):930-4. doi: 10.1136/jcp.2010.077867. PubMed PMID: 20876328.

98. Moon JH, Na JY, Kim JH, Yum MK, Oh JW, Kim CR, et al. Neurological and muscular manifestations associated with influenza B infection in children. Pediatr Neurol. 2013 Aug;49(2):97-101. doi: 10.1016/j.pediatrneurol.2013.04.004. PubMed PMID: 23859854.

99. Alonso WJ, Viboud C, Simonsen L, Hirano EW, Daufenbach LZ, Miller MA. Seasonality of influenza in Brazil: a traveling wave from the Amazon to the subtropics. Am J Epidemiol. 2007 June 15;165(12):1434-42. PubMed PMID: 17369609

100. Antonova EN, Rycroft CE, Ambrose CS, Heikkinen T, Principi N. Burden of paediatric influenza in Western Europe: a systematic review. BMC Public Health. 2012 Nov 12;12:968. doi: 10.1186/1471-2458-12-968. PMID: 23146107.

101. Karve S, Misurski DA, Meier G, Davis KL. Employer-incurred health care costs and productivity losses associated with influenza. Hum Vaccin Immunother 2013 Apr;9(4):841-57. doi: 10.4161/hv.23413. PubMed PMID: 23321849.

102. Prosser LA, Bridges CB, Uyeki TM, Hinrichsen VL, Meltzer MI, Molinari $N A$, et al. Health benefits, risks, and cost-effectiveness of influenza vaccination of children. Emerg Infect Dis. 2006 Oct;12(10):1548-58. doi: 10.3201/ eid1210.051015.

103. Dawood FS, Fiore A, Kamimoto L, Bramley A, Reingold A, Gershman K, et al. Burden of seasonal influenza hospitalization in children, United States, 2003 to 2008. J Pediatr. 2010 Nov;157(5):808-14.

104. Bender JM, Ampofo K, Gesteland P, Sheng X, Korgenski K, Raines B, et al. Influenza virus infection in infants less than three months of age. Pediatr Infect Dis J. 2010 Jan;29(1):6-9. doi: 10.1097/INF.0b013e3181b4b950. PubMed PMID: 19915513.

105. Bridges CB, Harper SA, Fukuda K, Uyeki TM, Cox NJ, Singleton JA. Prevention and control of influenza. Recommendations of the Advisory Committee on Immunization Practices (ACIP).MWR Recomm Rep. 2003 Apr 25;52(RR-8):1-34; quiz CE1-4. PubMed PMID: 12755288.

106. Chaves SS, Perez A, Farley MM, Miller L, Schaffner W, Lindegren ML, et al. The burden of influenza hospitalizations in infants from 2003-2012, United States. Pediatr Infect Dis J. 2014 Feb 26. PubMed PMID: 24577042.

107.Ampofo K, Gesteland PH, Bender J, Mills M, Daly J, Samore M, et al. Epidemiology, complications, and cost of hospitalization in children with laboratory-confirmed influenza infection. Pediatrics. 2006 Dec;118(6):2409-17. PubMed PMID: 714226.

108. Secretaria da Saúde (São Paulo, Brasil). Centro de Vigilância Epidemiológica. Informe técnico: situação epidemiológica da influenza, vigilância sentinela da influenza - Estado de São Paulo - Brasil [Internet]. São Paulo: Secretaria da Saúde ;2014 [atualizado 2014 Jan; citado 2014 Fev 7]. Disponível em: ftp://ftp. cve.saude.sp.gov.br/doc_tec/RESP/2014/IF14_INFLUJAN.pdf

109. Vaccines against influenza WHO position paper - November 2012. Wkly Epidemiol Rec. 2012 Nov 23;87(47):461-76. PubMed PMID: 23210147.

110. Bhat N, Wright JG, Broder KR, Murray EL, Greenberg ME, Glover MJ, et al. Influenza-associated deaths among children in the United States, 2003-2004. N Engl J Med. 2005 Dec 15;353(24):2559-67. PubMMed PMID: 16354892.

111. Hoen AG, Buckeridge DL, Charland KM, Mandl KD, Quach C, Brownstein JS. Effect of expanded US recommendations for seasonal influenza vaccination: comparison of two pediatric emergency departments in the United States and Canada. CMAJ. 2011 Sep 20;183(13):E1025-32. doi: 10.1503/cmaj.110241. PubMed PMID: 21930745.

112. Monto AS, Davenport FM, Napier JA, Francis T Jr. Modification of an outbreak of influenza in Tecumseh, Michigan by vaccination of schoolchildren. $J$ Infect Dis. 1970 July-Aug;122(1):16-25. PubMed PMID: 5433709.

113. Verani JR, McCracken J, Arvelo W, Estevez A, Lopez MR, Reyes L, et al. 
Surveillance for hospitalized acute respiratory infection in Guatemala. Plos One. 2013 Dec 31;8(12):e83600. doi: 10.1371/journal.pone.0083600. PubMed PMID: 24391792.

114. Ortega-Sanchez IR, Molinari NA, Fairbrother G, Szilagyi PG, Edwards KM, Griffin MR, et al. Indirect, out-of-pocket and medical costs from influenzarelated illness in young children. Vaccine. 2012 June 13;30(28):4175-81. doi: 10.1016/j.vaccine.2012.04.057. PubMed PMID: 22546332.

115. Kwong JC, Maaten S, Upshur RE, Patrick DM, Marra F. The effect of universal influenza immunization on antibiotic prescriptions: an ecological study. Clin Infect Dis. 2009 Sep 1;49(5):750-6. doi: 10.1086/605087. PubMed PMID: 19624280.

116. Nair H, Simões EA, Rudan I, Gessner BD, Azziz-Baumgartner E, Zhang JS, et al. Global and regional burden of hospital admissions for severe acute lower respiratory infections in young children in 2010: a systematic analysis. Lancet. 2013 Apr 20;381(9875):1380-90. doi: 10.1016/S0140-6736(12)61901-1. PubMEd PMID: 23369797.

117. Fairbrother G, Cassedy A, Ortega-Sanchez IR, Szilagyi PG, Edwards KM, Molinari NA, et al. High costs of influenza: direct medical costs of influenza disease in young children. Vaccine. 2010 July 12;28(31):4913-9. doi: 10.1016/j. vaccine.2010.05.036. PubMed PMID: 20576536

118. Keech $M$, Beardsworth P. The impact of influenza on working days lost: a review of the literature. Pharmacoeconomics. 2008;26(11):911-24. PubMed PMID: 1885761

119. Kostova D, Reed C, Finelli L, Cheng PY, Gargiullo PM, Shay DK, et al. Influenza illness and hospitalizations averted by influenza vaccination in the United States, 2005-2011. PLoS One. 2013 June 19;8(6):e66312. PubMed PMID: 23840439.

120. Marchisio P, Baggi E, Bianchini S, Principi N, Esposito S. Clinical and socioeconomic impact of pediatric seasonal and pandemic influenza. Hum Vaccin Immunother. 2012 Jan;8(1):17-20. doi: 10.4161/hv.8.1.18145. PubMed PMID: 22252002

121. Meltzer MI, Neuzil KM, Griffin MR, Fukuda K. An economic analysis of annual influenza vaccination of children. Vaccine. 2005 Jan 11;23(8):1004-14. PubMed PMID: 15620473

122. Molinari NA, Ortega-Sanchez IR, Messonnier ML, Thompson WW, Wortley PM, Weintraub E, et al. The annual impact of seasonal influenza in the US measuring disease burden and costs. Vaccine. 2007 June 28;25(27):5086-96. PubMed PMID: 17544181

123. Thors V, Smith C, Finn A. Should all children be immunised against influenza?. Arch Dis Child. 2013 Nov;98(11):846-9. PubMed PMID: 23908190

124. Weycker D, Edelsberg J, Halloran ME, Longini IM Jr, Nizam A, Ciuryla V, et al. Population-wide benefits of routine vaccination of children against influenza. Vaccine. 2005 Jan 26;23(10):1284-93. PubMed PMID: 15652671.

125. Reichert TA, Sugaya N, Fedson DS, Glezen WP, Simonsen L, Tashiro M. The Japanese experience with vaccinating schoolchildren against influenza. N Engl J Med. 2001 Mar 22;344(12):889-96. PubMed PMID: 11259722.

126. Mizumoto K, Yamamoto T, Nishiura H. Age-dependent estimates of the epidemiological impact of pandemic influenza (H1N1-2009) in Japan. Comput Math Methods Med. 2013;2013:637064. doi: 10.1155/2013/637064. PubMed PMID: 23509599.

127. Kjos SA, Irving SA, Meece JK, Belongia EA. Elementary school-based influenza vaccination: evaluating impact on respiratory illness absenteeism and laboratory-confirmed influenza. PloS One. 2013 Aug 26;8(8):e72243. doi: 10.1371/journal.pone.0072243. PubMed PMID: 23991071

128. Miller JR, Short VL, Wu HM, Waller K, Mead P, Kahn E, et al. Use of a nonpharmaceutical interventions to reduce transmission of 2009 pandemic influenza A (pH1N1) in Pennsylvania public schools. J Sch Health. 2013 Apr;83(4):281-9. doi: 10.1111/josh.12028. PubMed PMID: 23488889

129. Tashani M, Rashid H, Ridda I, Heron L, Memish ZA, Haworth E, et al. Oseltamivir for control of influenza at mass gatherings. Infect Disord Drug Targets. 2013 Feb;13(1):46-52. . PubMed PMID: 23675926.

130. Garza RC, Basurto-Dávila R, Ortega-Sanchez IR, Carlino LO, Meltzer $\mathrm{MI}$, Albalak R, et al. Effect of winter school breaks on influenza-like illness, Argentina, 2005-2008. Emerg Infect Dis. 2013 June;19(6):938-44. doi: 10.3201/ eid1906.120916. PubMed PMID: 23735682.

131. Sugisaki K, Seki N, Tanabe N, Saito R, Sasaki A, Sasaki S, et al. Effective school actions for mitigating seasonal influenza outbreaks in niigata, Japan. PloS ONE. 2013 Sep 10;8(9):e74716. doi: 10.1371/journal.pone.0074716. PubMed PMID: 24040329.

132. Allison MA, Guest-Warnick G, Nelson D, Pavia AT, Srivastava R, Gesteland PH, et al. Feasibility of elementary school children's use of hand gel and facemasks during influenza season. Influenza Other Respi Viruses. 2010 July;4(4):223-9. doi: 10.1111/j.1750-2659.2010.00142.x. PubMed PMID: 20836797.

133. Kann L, Kinchen S, Modzelski B, Sullivan M, Carr D, Zaza S, et al. ILI-related school dismissal monitoring system: an overview and assessment. Disaster Med Public Health Prep. 2012 June;6(2):104-12. doi: 10.1001/dmp.2012.13. PubMed PMID: 22700017.

134. Kim TH, Johnstone J, Loeb M. Vaccine herd effect. Scand J Infect Dis. 2011 Sep;43(9):683-9. doi: 10.3109/00365548.2011.582247. PubMed PMID: 21604922

135. Chi RC, Reiber GE, Lipsky BA, Boyko EJ, Neuzil KM. Influenza vaccination rates of children in households with high-risk adults. Public Health Rep. 2010 Mar-Apr;125(2):192-8. PubMed PMID: 20297745.

136. Domingues CM, de Oliveira WK, Brazilian Pandemic Influenza Vaccination Evaluation Team. Uptake of pandemic influenza (H1N1)-2009 vaccines in Brazil, 2010. Vaccine. 2012 July 6;30(32):4744-51. doi: 10.1016/j.vaccine.2012.05.007. PubMed PMID: 22609010

137. WHO. Questions and answers: recommended composition of influenza virus vaccines for use in the 2014 southern hemisphere influenza season [Internet]. 2013 [updated 2013 Jan; cited 2013 Sep 26]. Available from: http:// www.who.int/influenza/vaccines/virus/recommendations/201309_qanda recommendation.pdf

138. Belshe RB. The need for quadrivalent vaccine against seasonal influenza. Vaccine. 2010 Sep 7;28 Suppl 4:D45-53. doi: 10.1016/j.vaccine.2010.08.028. PubMed PMID: 2071360.

139. Bricks L, Carvalhanas TRMP, Paiva TM, Benega MA, Santos KCO, Silva DBB, Ferreira PM, et al. Influenza B circulation in Brazil and characterization of $75 \mathrm{~B}$ strains isolated from patients from São Paulo State, Brazil (2002-2013) [Internet]. In: 32nd Annual Meeting of the European Society for Paediatric Infectious Diseases; 2014 May 6-10; Dublin, Ireland [cited 2014 May 12]. Available from: http://espid.meetingxpert.net/ESPID_945/poster_94755/program.aspx

140. Paiva TM, Benega MA, Silva DB, Santos KC, Cruz AS, Hortenci MF, et al. Evolutionary pattern of reemerging influenza $\mathrm{B} /$ Victoria lineage viruses in São Paulo, Brazil, 1996-2012: implications for vaccine composition strategy. J Med Virol. 2013 Nov;85(11):1983-9. doi: 10.1002/jmv.23684. PubMed PMID: 23926069.

141. Oliveira MLA, Motta FC, Soares SS, Resende PC, Carney SM, Costa $J A C$, et al. Mismatch between vaccine strains and circulating influenza $b$ viruses in different regions of Brazil: 2001-2013. [Internet]. In: 8th Options for the Control of Influenza Conference; 2013 Sept 5-10; Cape Town, South Africa. Abstracts. 2013. p. 382-3. Available from: http://optionsviii. controlinfluenza.com/optionsviii/assets/File/Options_VIII_Abstracts_2013.pdf 